\title{
EL ESTUDIO DE LAS NECESIDADES Y EL DESARROLLO DE POLÍTICAS Y SERVICIOS A LAS FAMILIAS EN PUERTO RICO'
}

\author{
Dra. Diana Valle Ferrer, Catedrática Asociada
}

\section{Introducción}

$\mathbf{E}$ 1 bienestar social e individual ha sido identificado por algunos/as investigadores/as como el objetivo principal de las políticas sociales del Estado (Sapiro, 1990, Weale, 1983). La utilidad de esta definición estriba en su amplitud, pues incluye todas las facetas del bienestar general y no sólo la prestación de servicios sociales a los pobres o marginados. En el Estado capitalista, dice Pratts (1984), el propósito de la política social es mejorar la condición de vida en los componentes básicos como la nutrición, vivienda, salud, educación y otros. De acuerdo a Pratts (1984), su función es presentada ideológicamente como gestión del Estado en pro de la justicia social.

Por otro lado, Álvarez (1994) plantea que el "bienestar" puede ser uno entre diversos objetivos de las políticas del Estado y la importancia relativa que tenga dentro de las políticas depende del peso específico, del impacto y del alcance de tales políticas sobre el bienestar social y la calidad de vida de los diversos sectores sociales, regionales, de género, etc., en un determinado momento. La política social

\footnotetext{
${ }^{1}$ Una versión anterior de este trabajo fue escrito en colaboración con las profesoras Lilian Albite e Islia Rosado. El Estudio de necesidades para el apoyo y el fortalecimiento de las familias en Puerto Rico fue subvencionado por la Administración de Familias y Niños del Departamento de la Familia y el Omnibus Reconciliation Act de 1993.
} 
también se vincula con las necesidades humanas que no son satisfechas adecuadamente por la familia, la comunidad o el mercado. Las necesidades humanas y sociales han sido definidas como "aquellas condiciones o requerimientos materiales, sociales o espirituales indispensables para que los individuos y las comunidades puedan desplegar sus energías y capacidades creativas y de disfrute de la vida" (Álvarez; 1994:21).

Nos preguntamos entonces: si las políticas sociales en Puerto Rico responden a las necesidades de nuestras familias - mujeres, hombres, jóvenes y niños/as - y de nuestras comunidades, en el contexto socioeconómico de hoy, ¿quién o quiénes deben satisfacer las necesidades de nuestras familias?; ¿el gobierno, el mercado, las propias familias?. Son unas pregunta que tienen diferentes contestaciones dependiendo del análisis teórico e ideológico que se haga. Está también en cuestionamiento cuáles son estas necesidades, y más profundamente nos cuestionamos el concepto mismo de necesidades y de familia. ¿Hablamos de la familia tradicional nuclear que surge como producto de la industrialización basada en el "hombre jefe de familia" que tiene un trabajo con suficientes ingresos para "mantener" y ser "proveedor" de "su familia"? ¿O estamos hablando de la diversidad de familias que existen hoy día, por ejemplo una mujer con sus hijos, parejas lesbianas y homosexuales criando hijos o hijas de uno o de ambos, o una abuela con sus nietos/as, etc.? ¿Cuando hablamos sobre las necesidades de "la familia" nos referimos a las necesidades de la mujer, de los jóvenes o de los niños en sus familias? En todas estas controversias se disputa quiénes exactamente componen estas familias, qué realmente necesitan para lograr su bienestar y quién o quiénes lo definen.

Además de los debates de paradigmas, el trabajo social se ha visto inmerso en un mar de transformaciones económicas y de política social cuya trama ha sido hilvanada por otros actores sociales y que tienen una inmediata repercusión en las poblaciones para quienes trabaja. A modo de síntesis de este abanico de transformaciones podemos mencionar la avanzada neoliberal que se inicia en la década de los 70 y que se manifiesta en el retorno de prácticas del primer capitalismo del siglo pasado, en que se da la preferencia al mercado como principal mecanismo para la solución de los problemas sociales 
y del estancamiento económico (Valle, Albite, Rosado, 1995).

Esta tendencia neoliberal plantea además la reducción del aparato estatal mediante la ampliación de los mecanismos de privatización; limitaciones al gasto social, mayormente en las políticas de bienestar social; surge la desreglamentación que elimina subsidios, servicios y beneficios a las clases marginadas y se acelera la reducción de empleos tanto en el gobierno como en el sector privado. Ante estas medidas se produce el debilitamiento de la sociedad civil en general y de la clase trabajadora y los pobres en particular. Por otro lado, se producen modelos alternativos que se caracterizan por el énfasis en la capacidad de autoresolución y autorganización de las personas frente a las necesidades sociales (Estivill, 1985). Estos modelos tienen como finalidad la liberación individual y colectiva y la promoción de la participación y autogestión de los usuarios de los servicios sociales.

En este nuevo reordenamiento y reajuste a las reglas del juego no todo está escrito y dicho en forma final. En este reajuste neoliberal, aunque a los trabajadores sociales no nos guste, se entra en un proceso de experimentación y reorganización de las fuerzas tanto en el sector público como en la sociedad civil. Se reconoce el peligro inminente para los sectores marginados, pero conjuntamente se crea la conciencia de una nueva oportunidad de renovar la acción política. La redefinición del papel que juega el Estado en cuanto a su compromiso social nos ofrece la oportunidad de participar en su reestructuración. Surge, entonces, la necesidad de trabajar para crear nuevos modelos de desarrollo económico, la autogestión, la cogestión, rescatar y hacer valer la política de derechos humanos y replantearse lo que significa la democracia, la igualdad y la solidaridad.

Ante este panorama los/as trabajadores/as sociales no tenemos más remedio que afrontar nuestra responsabilidad de acción política y preguntarnos: ¿Qué podemos hacer en la práctica profesional a fin de que, en cumplimiento con nuestro compromiso ético, los nuevos arreglos sociales más bien favorezcan y no dificulten más, un proyecto de una nueva sociedad más justa para todos y todas? ¿Qué función corresponde al Trabajo Social que actualmente conocemos frente a la aspiración de las clases marginadas por construir una realidad cualitativamente más humana y plena en esta sociedad tan compleja, 
llena de contradicciones e incertidumbre? ¿Desde qué vertientes y en qué forma puede hacer posible su contribución?

Para contestar estas preguntas, es evidente que muchas de las estrategias tradicionales ya no operan en este nuevo estado de situación. Por lo tanto, el trabajo social debe revisar cuidadosamente sus marcos teóricos, construir nuevas formas para su quehacer práctico y vigilar que su compromiso ético guíe su ejecutoria en cualesquiera de los escenarios donde trabaje. Con ello queremos decir que en este momento la práctica del trabajo social, no importa su escenario y nivel de práctica (servicio directo, administración, investigación, docencia, consultoría y acción social) es un trabajo de acción política. Esto es así en tanto la forma y contenidos que asumamos en nuestra práctica profesional constituyan de por sí una contribución, querámoslo o no, al reordenamiento de las reglas del juego que se está dando. Partiendo de esta óptica tenemos que abandonar la seguridad de lo obvio y ortodoxo para replantearnos qué impacto tiene nuestro quehacer en la vida de los usuarios de los servicios que ofrecemos.

Desde esta perspectiva amplia de la práctica profesional y la acción política es que hemos enmarcado nuestro trabajo. La autora presenta cómo en el contexto de facilitar la implantación de una nueva medida de política social en Puerto Rico se pueden rescatar los componentes de justicia social y de vinculación social comunitaria cuya fuerza está en la cogestión entre la sociedad civil con las Organizaciones no Gubernamentales (ONG) y el gobierno. Durante el año 1994-95 tuvimos la oportunidad de llevar a cabo un amplio estudio de las necesidades de las familias en Puerto Rico y de elaborar a partir de sus hallazgos un Plan Estratégico de Cinco Años (Valle, Albite, Rosado; 1995) para el desarrollo de un programa de Servicios de Apoyo y Fortalecimiento de las Familias en Puerto Rico.

Al tener a cargo esta importante tarea iniciamos una reflexión sobre las formas y contenidos de nuestro quehacer para articular nuestra contribución de acción política en un esquema conceptual a tono con un posible proyecto para una sociedad diferente. Nuestra primera hipótesis de trabajo fue la premisa de que entre el Estado y las personas sigue existiendo la dimensión social e histórica de compromiso de uno con el otro. Debido a esto, las transformaciones del 
Estado no pueden separarse del fortalecimiento de los vínculos sociales comunitarios y de propuestas de trabajo democratizantes. A partir de aquí teníamos ya que revisar nuestras conceptualizaciones y volvernos a preguntar: ¿Qué entendemos por necesidades? ¿Quiénes las definen? ¿Cómo se han articulado desde la perspectiva política? ¿Cómo queremos que sea esta noción? ¿Qué es una familia? ¿Cómo se ha representado políticamente? ¿Cómo debería representarse para un proyecto más democratizante? ¿Cómo desarrollamos un estudio de necesidades que recoja las voces de la gente dentro de los recursos y posibilidades existentes? Y, ¿cómo articulamos un Plan Estratégico de Cinco Años para el Apoyo y Fortalecimiento de la Familia que devuelva a la sociedad civil un poder para articular sus propias alternativas de servicio en las comunidades, centradas en las familias, desde las perspectivas de las familias y en cogestión y auspicio por el Estado? (Valle, Albite, Rosado; 1995).

A continuación intentaré, en forma sucinta, recoger todo el esfuerzo por contestar en un proyecto concreto todas las preguntas antes descritas y articular en un esquema diferente un modelo de estudio de necesidades y la aplicación de sus hallazgos a un programa de política social.

\section{Trasfondo general de la legislación a implantarse en Puerto Rico}

En el año 1993 se aprobó una nueva política federal mejor conocida como el Omnibus Reconciliation Act cuyo objetivo principal era ampliar los esfuerzos de prevención y mejorar los servicios de apoyo a las familias, particularmente de aquellas denominadas en alto riesgo, de manera que se propiciara en el seno de la familia un ambiente más seguro para el desarrollo de todos sus miembros. A tales efectos, se proveyeron fondos a todos los estados y a Puerto Rico para que se evaluaran los servicios existentes, se identificaran las necesidades de las familias y se efectuaran cambios en la forma en que se prestan los servicios. Esta legislación deja abierta la definición de familia que incluye todo tipo de estructuras familiares. Se pretendía establecer un ofrecimiento continuo de servicios integrados cuyo foco de atención fueran los menores y sus familias, y que dichos servicios se prestaran en las mismas comunidades. 
Los objetivos generales de la legislación son:

- Salvaguardar la seguridad de todos los miembros de la familia.

- Fortalecer las habilidades de la familia para promover el desarrollo saludable de sus hijos e hijas.

- Contribuir al desarrollo de servicios para las familias y sus hijos que sean verdaderamente efectivos, accesibles y de acuerdo con sus necesidades.

Durante el año 1994-95 las investigadoras (Valle, Albite, Rosado; 1995) y consultoras a cargo de la implantación de este proyecto llevaron a cabo un estudio de necesidades para sentar las bases de la planificación. Teniendo en mente todas las preguntas antes mencionadas nos dimos a la tarea de detenernos a revisar la conceptualización de un estudio de necesidades y la noción misma de necesidad.

\section{El estudio de necesidades y la noción de necesidad:}

\section{La articulación de un modelo de orientación y}

\section{vinculación comunitaria}

Los estudios de necesidades surgieron asociados a la creación del estado benefactor y el capitalismo regulado (Lechner, 1988; Fraser, 1989). A partir de la década de los 30 el Estado inicia intervenciones a través de políticas públicas fiscales, monetarias y sociales en el interés de encauzar la actividad económica a la vez que se creaban programas y ayudas sociales para atender las demandas de los sectores marginados por la economía. Se trataba así de mantener un orden social, estabilidad y tener niveles mínimos de distribución de beneficios sociales (Pratts, 1994). Es aquí que las necesidades humanas entran a la arena del discurso político ante la determinación del Estado de crear modelos de programas sociales. Surge toda una política de interpretación de necesidades con un análisis mediatizado por diversos factores: la validación o no de la necesidad a nivel político, quién en última instancia la define y la lucha para que la misma se satisfaga. Surge, por tanto, todo un idioma de necesidades, una tecnología social científica para su identificación, y una diversidad de paradigma e ideologías para el reclamo de su satisfacción. 
En la dimensión tecnológica el análisis de necesidades se convierte en una metodología de ciencia aplicada imbricada en los procesos de planificación. El estudio de necesidades conlleva la identificación y evaluación de necesidades para diversos propósitos usualmente ligados, entre otras, a la toma de decisiones relacionadas con la distribución de recursos, delimitar prioridades para la acción, justificar una labor y diseño de servicios y programas (Meenagham $\&$ Keith, 1994). Se incorpora esta tecnología a los muy conocidos modelos de desarrollo y a toda práctica de programación de servicios.

En la revisión de la literatura se encuentra una gran diversidad sobre quién y desde qué perspectiva se definen las necesidades. La literatura tiende a sugerir que la definición de la noción de necesidad responde en última instancia a aquellos que asumen la tarea de su identificación. No obstante, diversos autores coinciden que los modelos de los programas sociales se montaron sobre discursos construidos sobre nociones terapéuticas de los peritos de la conducta, en que las necesidades eran principalmente responsabilidad individual, nociones moralistas, religiosas y paternalistas, criterios económicos y criterios administrativos (Fraser, 1989). Un ejemplo de esta conceptualización puede incluir desde la visualización de las necesidades de las mujeres, no como personas, sino como esposas y madres, supeditadas a los roles familiares desde perspectivas paternalistas y moralistas.

Ya a partir de la década de los años 60 surge una pluralidad de reclamos de grupos sociales minoritarios con desigualdad de poder y acceso a los recursos y beneficios sociales, entre ellos las mujeres, que replantea las necesidades como derechos. Estos grupos a su vez tienen una amalgama de reclamos tan diversos como lo son también los sectores que las proclaman. A la larga estos reclamos no tienen un demarcamiento consolidado. De aquí surge un sector de expertos que se han dado a la tarea de conectar estos reclamos con el estado.

El Estudio de Necesidades de las Familias en Puerto Rico (Valle, Albite, Rosado; 1995) se llevó a cabo y se articuló desde la noción de necesidad como derecho, así como también la del respeto a la diversidad. Esto último relacionado principalmente con el punto de partida de una definición de familia construida propiamente por la gente y no por los peritos. De aquí que "fámilias" podía incluir todo tipo de 
arreglo de relaciones significativas e íntimas que enmarcara para los participantes su propia construcción de lo que es familia. Las voces de estos actores sociales iba a construir el criterio principal para la interpretación de los hallazgos, la priorización y jerarquización de las necesidades.

Enmarcado en los principios de trabajo antes mencionados, se plantea, para propósitos de este estudio, la necesidad como una discrepancia o vacío existente entre lo que se considera una condición aceptable o deseable (según los participantes) y el estado actual existente. Este enfoque de discrepancia trasciende el término de necesidad como uno definido desde la perspectiva individualista a uno de análisis situacional/estructural que recoge el binomio personaambiente ampliándose así los elementos de análisis. Este binomio recoge a su vez una mayor diversidad de actores sociales que pueden contribuir al análisis de la situación de las familias. Así pues, los grupos afectados - en este caso familias, mujeres y niños/as - así como también los "expertos" - profesionales de ayuda, proveedores de servicio, líderes comunitarios y otros observadores de la comunidad en general - se añaden a los grupos específicos de consulta (Valle, Albite, Rosado; 1995).

En la configuración de las estrategias metodológicas se hizo hincapié en la diversificación de las fuentes de información cuantitativa y cualitativa de manera que se pudiera llevar a cabo un análisis desde una perspectiva más amplia del problema. El enfoque fue desarrollado con una metodología interactiva desde una perspectiva ecológica y orientada a la comunidad. Se utilizó la base teórica de la teoría de sistemas sociales en que el enfoque ecológico alienta al investigador/a a buscar la interrelación entre las variables a todos los niveles de influencia en los problemas que enfrenta la familia puertorriqueña. Este acercamiento teórico está de acuerdo con nuestro análisis y el concepto de persona ambiente. A tales efectos teníamos que estar sensitivos a cómo el micro sistema de la familia se nutre, afecta y es afectado por los sistemas medianos y grandes que le rodean. A tales efectos era necesario recoger información de todos los actores - en primer lugar la familia y también los líderes comunitarios, profesionales de ayuda, organizaciones (información testimonial)—, así como también datos numéricos de indicadores sociales 
tomados de varias fuentes (compilación estadistica y numérica de varias fuentes). Estos indicadores nos permiten tener un cuadro más amplio de una comunidad o un área geográfica en particular. La orientación comunitaria se destacó con la incorporación al modelo y el insumo de las familias desde las propias comunidades, en que éstas constituían el eje central de la consulta.

A tono con el diseño antes descrito, se desarrolla un modelo para el estudio de necesidades (Valle, Albite, Rosado; 1994). El modelo se construyó en el contexto de las siguientes estrategias o técnicas metodológicas:

\section{Fuentes primarias}

1. TÉCnICA DE GRUPos Focales - Dieciséis grupos focales en que participaron familias, adolescentes, jóvenes transgresores de la ley, sindicatos obreros, líderes de la comunidad, proveedores de servicios públicos y privados y profesionales de ayuda. Los grupos se celebraron en el norte, sur, este, oeste y zona central de la isla.

2. VISTAS Públicas - Diecisiete vistas públicas en comunidades por las distintas zonas de la isla en las que participaron destacadamente las familias en sesiones de diálogo al igual que líderes comunitarios y representantes de ONG.

3. ENCUESTAS DE OPINIÓN - Tres (3) encuestas en que participaron las familias, las ONG y el personal de servicio directo a las familias del Departamento de la Familia.

\section{Fuentes secundarias}

4. ANÁlisis de IndiCAdores Sociales - Censo de Puerto Rico y estudios realizados en Puerto Rico.

5. INVENTARIO PRELIMINAR DE RECURSOS - Especialmente dirigido a identificar las ONG que dan servicios a las familias.

6. ANÁLISIS DE INFORMACIÓN SOBRE LOS PARTICIPANTES DE LOS SERVICIOS QUE OFRECE EL DEPARTAMENTO DE LA FAMILIA • Datos disponibles de los Programas de Familias y Niños, Asistencia Nutricional, Protección a Menores, Asistencia Económica. 


\section{Utilización de los hallazgos y la tarea de planificación de política social}

Dada la magnitud de este estudio y la imposibilidad de articular en tan poco espacio la riqueza de los hallazgos recogidos en informes extensos (vistas públicas, grupos focales, encuestas, análisis demográfico y el informe del estudio integrado) nos limitaremos a resumir en las Tablas 1, 2 y 3 algunos indicadores sociales sobre la familia y las necesidades según expresadas por todos los participantes en las vistas públicas, grupos focales y encuestas, por orden de rango. $\mathrm{Pa}$ saremos a señalar lo que a nuestro juicio han sido los aspectos concluyentes y sobre todo a destacar el proceso de la utilización de los hallazgos para establecer una política de vinculación comunitaria y cogestión.

La Tabla 1 recoge algunos de los indicadores sociales sobre las familias en Puerto Rico. De acuerdo con estos datos, dos de los factores más significativos que afectan a las familias en Puerto Rico son la pobreza, especialmente entre las familias dirigidas por mujeres, y la falta de protección y acceso a la educación de niños/as y jóvenes.

La Tabla 2 refleja una preocupación de los participantes en las vistas públicas y los grupos focales con las necesidades de la niñez y los adolescentes. Estas necesidades están relacionadas con la falta de supervisión, cuidado adecuado y actividades fuera del horario escolar de parte de sus padres, del gobierno y la comunidad. Además, en la Tabla 2 se refleja una preocupación de los participantes con la falta de apoyo para madres jefas de familia, sobrevivientes de violencia doméstica y para personas envejecientes. Otros factores de preocupación son la falta de empleo, el aumento en la incidencia de violencia en la familia, embarazo en adolescentes y uso y abuso de drogas y alcohol.

De la Tabla 3 se desprende que las necesidades más apremiantes para las familias fueron empleo, ayuda económica y vivienda. El personal de servicio directo del Departamento de la Familia opinó que la vivienda y el empleo son necesidades urgentes de las familias (rango 2 y 3 respectivamente). El personal de organizaciones sin fines de lucro también señaló la necesidad de empleo pero le dieron un sexto lugar en el orden de rango. 
Tabla 1

Indicadores de necesidades de apoyo

$y$ fortalecimiento de la familia

\begin{tabular}{lll}
\hline \multicolumn{1}{c}{ Indicadores } & Unidad de medición & \multicolumn{1}{c}{ Fuente } \\
\hline Niños/as & & \\
\hline Pobreza & $\begin{array}{l}\text { Uno de cada cinco (1/5) vive } \\
\text { en extrema pobreza }\end{array}$ & $\begin{array}{l}\text { Est. Necesidades / } \\
\text { ASSMCA Resources, Inc. }\end{array}$ \\
\hline $\begin{array}{l}\text { Familias con niños/as } \\
\text { que reciben asistencia } \\
\text { económica }\end{array}$ & $\begin{array}{l}54,679 \text { Familias 5.9\% del } \\
\text { total de familias en P.R. }\end{array}$ & $\begin{array}{l}\text { Departamento de la Familia, } \\
1995\end{array}$ \\
\hline $\begin{array}{l}\text { Protección de menores } \\
\text { Referidos }\end{array}$ & 27,042 familias (1993-94) & $\begin{array}{l}\text { Planif. y Desarrollo, 1995 } \\
\text { Departamento de la Familia }\end{array}$ \\
\hline $\begin{array}{l}\text { Protección de menores } \\
\text { Casos }\end{array}$ & 15,575 familias (1993-94) & $\begin{array}{l}\text { Planif. y Desarrollo, 1995 } \\
\text { Departamento de la Familia }\end{array}$ \\
\hline
\end{tabular}

\section{Adolescentes}

\begin{tabular}{|c|c|c|}
\hline Activo sexualmente & $\begin{array}{l}56.7 \% \text { de la edad de } 15 \\
92.2 \% \text { de la edad de } 17\end{array}$ & Departamento de Educación \\
\hline Embarazo en adolescentes & 1 de cada 5 nacimientos & $\begin{array}{l}\text { Departamento de Salud, } \\
1985\end{array}$ \\
\hline Aprovechamiento Escolar & $\begin{array}{l}40 \% \text { de los que entran a } \\
1 \text { er. grado no se gradúa } \\
55 \% \text { fracasa esc. elemental } \\
30 \% \text { fracasa esc. intermedia } \\
16 \% \text { fracasa esc. superior }\end{array}$ & $\begin{array}{l}\text { Vale, } 1980 \\
\text { Depto. Educación, } 1985\end{array}$ \\
\hline
\end{tabular}

\section{Familias}

\begin{tabular}{lll}
\hline Mujeres jefas de familia & 23\% del total de familias & Censo, 1990 \\
\hline Familias de un solo jefe & $\begin{array}{l}\text { 1 de cada 10 familias en P.R. } \\
\text { 4 de cada 10 familias } \\
\text { en residenciales }\end{array}$ & $\begin{array}{l}\text { Censo, 1990 } \\
\text { Estudio de necesidades } \\
\text { ASSMCA-Resources, Inc. }\end{array}$ \\
\hline $\begin{array}{l}\text { Familias que reciben } \\
\text { Asistencia Nutricional (PAN) }\end{array}$ & $\begin{array}{l}485,459 \text { o el 39\% de } \\
\text { la población }\end{array}$ & $\begin{array}{l}\text { Planif. y Desarrollo, Depto. } \\
\text { de la Familia, 1995 }\end{array}$ \\
\hline $\begin{array}{l}\text { Familias que reciben } \\
\text { Asistencia Económica (AE) }\end{array}$ & $\begin{array}{l}97,553 \text { familias o el 11\% de } \\
\text { todas las familias }\end{array}$ & $\begin{array}{l}\text { Planif. y Desarrollo, Depto. } \\
\text { de la Familia, 1995 }\end{array}$ \\
\hline
\end{tabular}

Preparado por C.O.S.E.P., Inc. Proyecto de Apoyo a la Familia 
Tabla 2

Lista de necesidades y preocupaciones expresadas por los participantes en las vistas públicas y los grupos focales en orden de rango

\begin{tabular}{l} 
Vistas públicas \\
\hline 1. Necesidad de reducir los \\
embarazos. \\
2. Los padres necesitan más \\
destrezas para criar a sus \\
hijos/as. \\
3. Necesidad de aumentar los \\
servicios de apoyo a las mujeres \\
jefas de familia.
\end{tabular}

4. Alta incidencia de desempleo.

5. Los jóvenes abandonan la escuela a temprana edad.

6. Las familias tienen pocas oportunidades de recreación en las que participan todos los miembros.

7. Falta de actividades para los adolescentes después del horario escolar.

8. Necesidad de aumentar los servicios de amas de llave.

9. Necesidad de grupos de apoyo en las comunidades para ayudar a las familias en situaciones de conflicto y crisis.

10. Aumento en los problemas de comunicación entre padres e hijos/as.

11. Aumento en la incidencia de violencia en la familia.

\section{Grupos focales}

1. Ocio en la población adolescente /falta de actividades después del horario escolar.

2. Aumento en la deserción escolar.

3. Necesidad de orientación y consejería a jóvenes sobre uso y abuso de alcohol y drogas; educación sexual.

4. Falta de grupos de apoyos para los hijos e hijas de mujeres sobrevivientes de violencia doméstica.

5. Distanciamiento en la comunicación entre padres y madres e hijos/as.

6. Escasez de oportunidades de empleo.

7. Servicios de cuidado infantil que permitan a la mujer trabajar asalariadamente fuera del hogar.

8. Orientación para adolescentes embarazadas.

9. Orientación y consejería sobre crianza de hijos/as y prevención de maltrato y negligencia.

10. Necesidad de aumentar los servicios de ama de llave.

11. Más programas de prevención de violencia familiar. 


\section{Tabla 3}

Lista de necesidades y preocupaciones en orden de rango y según expresadas por las familias, personal del servicio directo $\mathrm{y}$ organizaciones que participaron en las encuestas

\begin{tabular}{|c|c|c|}
\hline \multicolumn{3}{|c|}{ Personal de servicio Organizaciones } \\
\hline $\begin{array}{l}\text { 1. Oportunidad de } \\
\text { empleo }\end{array}$ & $\begin{array}{l}\text { 1. Orientación a los } \\
\text { padres sobre la } \\
\text { crianza de los/as } \\
\text { hijos/as }\end{array}$ & $\begin{array}{l}\text { 1. Orientación sobre } \\
\text { uso y abuso de } \\
\text { drogas }\end{array}$ \\
\hline 2. Ayuda económica & 2. Vivienda & $\begin{array}{l}\text { 2. Incrementar la } \\
\text { asistencia para } \\
\text { víctimas de maltra- } \\
\text { to a menores }\end{array}$ \\
\hline 3. Vivienda & $\begin{array}{l}\text { 3. Oportunidades de } \\
\text { empleo }\end{array}$ & 3. Transportación \\
\hline $\begin{array}{l}\text { 4. Disponibilidad de } \\
\text { medicinas }\end{array}$ & $\begin{array}{l}\text { 4. Aumento en los } \\
\text { servicios de salud } \\
\text { mental }\end{array}$ & $\begin{array}{l}\text { 4. Aumentar los } \\
\text { servicios médicos }\end{array}$ \\
\hline $\begin{array}{l}\text { 5. Accesibilidad a } \\
\text { servicios de los } \\
\text { servicios de salud }\end{array}$ & $\begin{array}{l}\text { 5. Accesibilidad a los } \\
\text { servicios de salud }\end{array}$ & $\begin{array}{l}\text { 5. Asistencia Econó- } \\
\text { mica }\end{array}$ \\
\hline $\begin{array}{l}\text { 6. Recreación para } \\
\text { toda la familia }\end{array}$ & $\begin{array}{l}\text { 6. Mejorar la nutrición } \\
\text { infantil }\end{array}$ & $\begin{array}{l}\text { 6. Oportunidades de } \\
\text { empleo }\end{array}$ \\
\hline 7. Transportación & $\begin{array}{l}\text { 7. Servicios de } \\
\text { cuidado infantil }\end{array}$ & $\begin{array}{l}\text { 7. Programas de } \\
\text { prevención para la } \\
\text { juventud (violencia, } \\
\text { maltrato) }\end{array}$ \\
\hline $\begin{array}{l}\text { 8. Cuidado de niños/ } \\
\text { as que le permita a } \\
\text { las mujeres trabajar } \\
\text { fuera del hogar }\end{array}$ & $\begin{array}{l}\text { 8. Educación pre- } \\
\text { escolar }\end{array}$ & $\begin{array}{l}\text { 8. Asistencia a las } \\
\text { familias en estado } \\
\text { de crisis }\end{array}$ \\
\hline $\begin{array}{l}\text { 9. Educación vocacio- } \\
\text { nal }\end{array}$ & $\begin{array}{l}\text { 9. Actividades } \\
\text { recreativas }\end{array}$ & $\begin{array}{l}\text { 9. Aumentar las } \\
\text { facilidades recreati- } \\
\text { vas en las comuni- } \\
\text { dades }\end{array}$ \\
\hline $\begin{array}{l}\text { 10. Aumentar los } \\
\text { servicios de salud } \\
\text { mental }\end{array}$ & $\begin{array}{l}\text { 10. Programas de } \\
\text { educación vocacio- } \\
\text { nal }\end{array}$ & \\
\hline
\end{tabular}


Se puede observar con respecto a la mayoría de las necesidades que los resultados no fueron consistentes en términos de la preocupación de las familias participantes vis a vis el personal de ayuda. El personal de ayuda (servicio directo y organizaciones) señaló como necesidades apremiantes el apoyo emocional y la orientación, sin descartar la necesidad imperiosa de empleo, ayuda económica y vivienda. Las familias, por otro lado, entienden que sus necesidades más urgentes son de naturaleza estructural y social.

De los hallazgos que se reflejan en las Tablas 1,2 y 3 podemos destacar las siguientes conclusiones generales:

1. Las familias en nuestras comunidades experimentan unas condiciones de precariedad originadas por la falta de empleo, acceso a servicios de salud adecuados y carencia de apoyo para criar a sus hijos e hijas en sus comunidades.

2. Si bien los profesionales de ayuda reconocen las necesidades concretas de las familias, en muchas ocasiones ponen primero los enfoques terapéuticos individuales sobre las necesidades concretas que responden a problemas estructurales.

3. Se reconoce a las adolescentes embarazadas, las mujeres jefas de familia, las mujeres sobrevivientes de violencia y a los jóvenes de ambos sexos como las poblaciones de mayor necesidad.

4. Las familias en las comunidades están ávidas de tener oportunidades para enriquecer sus vidas y la de sus hijos e hijas en actividades familiares, recreativas, culturales y sociales - que son prácticamente inexistentes - para fomentar la solidaridad familiar y comunal.

5. Las familias en general y en particular las mujeres con jefatura de familia necesitan más oportunidades de cuido de niños y transportación para completar adiestramiento y obtener un empleo.

6. Es necesario promover la seguridad de los integrantes de la familia, especialmente mujeres, jóvenes y niños víctimas/sobrevivientes de violencia, lo que requiere además de los apoyos materiales externos, la ayuda de grupos de apoyo y de desarrollo 
de destrezas para la crianza.

7. Los jóvenes de ambos sexos necesitan vivir una vida libre de violencia y tener oportunidades para desplegar su creatividad y deseos de contribuir a su comunidad.

Como dato importante de nuestra experiencia en las comunidades tenemos que destacar que dentro de la situación de precariedad y marginación de las estructuras económicas, las familias consultadas sienten que sus fortalezas son ellas mismas. Se perciben como sobrevivientes gracias al apoyo entre los miembros de la familia y la iglesia, y todavía tienen fe en los profesionales de ayuda que están más cerca de ellos. Presentan una conciencia clara de sus necesidades (aunque no necesariamente las entienden como derechos) y están deseosos de participar creativamente en la reconstrucción de sus comunidades.

El proceso se diseñó a partir de estos hallazgos y en rescate de estas fortalezas de las comunidades. Se trabajó en sentar las pautas y principios ideológicos de la política de Apoyo y Fortalecimiento de la Familia. Con una junta consultiva compuesta por representantes de los distintos sectores sociales - líderes de la comunidad, familias, profesionales de ayuda, ONG y agencias de gobierno- se analizaron los hallazgos y se elaboró una visión y unos principios rectores para el diseño de los servicios de apoyo y fortalecimiento de las familias que, enmarcado en todo el análisis teórico e ideológico aquí descrito, sienta las pautas para una nueva y democratizadora política de familia. La visión y los principios rectores son los siguientes:

\section{LA VISIÓN}

Aspiramos a que nuestras familias sean fuertes y trabajen en conjunto dentro de sus comunidades. Deseamos que todos los miembros de la familia, con énfasis en los niños y niñas, los jóvenes y las mujeres, estén seguros y libres de violencia para desarrollarse plenamente. Concebimos los servicios de apoyo y fortalecimiento centralizados en la familia, con una amplia base comunitaria y con el compromiso y colaboración entre agencias públicas y privadas. Consideramos que los servicios deben ser abarcadores y accesibles tomando en cuenta las fortalezas, necesidades y diferencia de las familias y las comunidades. 


\section{PRINCIPIOS RECTORES}

Esta Visión requiere que los servicios:

- Promuevan la equidad, seguridad y bienestar de todos los miembros de familia con énfasis en los niños/as, los jóvenes y las familias.

- Apoyen a las familias en el proceso de lograr su autonomía y autosuficiencia.

- Enfaticen la prevención, reconozcan las fortalezas espirituales y físicas de las familias, así como sus aspiraciones y necesidades, y que se construya sobre esta base.

- Provean continuidad de los servicios, coordinados y diseñados en colaboración con las familias y otros programas existentes.

- Reconozcan a las familias, con énfasis en los niños y niñas, jóvenes y mujeres, como participantes activos y valiosos en la determinación y satisfacción de las necesidades de su comunidad.

- Apoyen a todas las familias en la comunidad para promover el desarrollo integral de niños y niñas, jóvenes y mujeres.

Estos principios establecen que todos los servicios estarán centrados en las familias, con y desde la perspectiva de la familia, en una relación de mayor igualdad entre sus miembros y ofrecido desde las propias comunidades. Se parte de las fortalezas de la familia y no de sus déficit y se expresa el compromiso del gobierno de apoyar a las familias en sus esfuerzos de tener una mejor calidad de vida.

A partir de esta articulación de misión se establece una planificación estratégica que se delimita a partir de los fondos que dispone la ley. La determinación de prioridades de trabajo en ese mar de necesidades y las áreas geográficas en que se comenzarían los esfuerzos constituyeron elementos decisionales de la planificación. A tales efectos, se procedió a recoger como prioridades aquellas necesidades que se suscitaron con más frecuencia en todas las instancias de consulta e investigación. Las necesidades expresadas por los participantes en el proceso de consulta planteaban problemas específicos como: desempleo y dependencia económica, embarazo en adolescentes, violencia contra la mujer, maltrato y negligencia de niños y niñas, necesidad de apoyo a las mujeres jefas de familia. 
Como dentro de los parámetros de la legislación del Omnibus Reconciliation Act de 1993 (Family Support and Family Prevention Act) no estaba el promover cambios en las estructuras económicas, ni la promoción de empleos, se decidió, como estrategia, promover la colaboración entre las agencias públicas y las ONG. Esto se hizo con el propósito de propiciar proyectos de autogestión y cogestión y así poder responder, aunque fuera en forma muy limitada, a la necesidad de empleo planteada por las mujeres y los/as jóvenes consultados. Una de las áreas que tendremos que evaluar posteriormente es si los empleos generados u obtenidos como resultados de los Proyectos de Apoyo a las Familias son de subsistencia o, si por el contrario, sacan a las mujeres y a los jóvenes de la pobreza y la dependencia. Ingresos de subsistencia mantienen a las mujeres y a las jóvenes subordinadas en sus familias y en sus comunidades, y tal vez sólo contribuyan a "sacarlos" de las listas del desempleo o de las de asistencia económica o nutricional.

En el Plan Estratégico de Cinco Años se desarrollaron objetivos específicos para responder a las necesidades identificadas por los diferentes sectores poblacionales, tales como mujeres, jóvenes y niños y niñas y en las diversas regiones que tenían la incidencia más alta de problemas en las áreas antes mencionadas. Por lo tanto, para establecer prioridades de planificación se utilizaron las estadísticas disponibles por municipio en estas áreas de problemas, como indicadores para seleccionar las comunidades de mayor necesidad de servicios de apoyo y fortalecimiento de la familia. Este análisis estadístico indicó que de un total de 72 municipios, doce, o el 16.6\%, tienen la incidencia más alta de tres o más indicadores. Estas áreas correspondían a seis regiones del Departamento de la Familia: Humacao, Mayagüez, Guayama, Caguas, Ponce y Carolina. De aquí que estas regiones constituyeron la prioridad inmediata para el desarrollo de servicios de apoyo y fortalecimiento de la familia.

Una convocatoria para someter propuestas a las ONG que deseaban prestar servicios enmarcados en la visión y principios rectores y a tono con las prioridades identificadas fue enunciada. A ella respondieron 81 organizaciones de la comunidad de las que se seleccionaron finalmente 41 . Los proyectos fueron subvencionados por el Estado, en particular el Departamento de la Familia, y reciben además la 
ayuda técnica, adiestramiento y asesoría en las áreas que ellos han identificado como sus necesidades para articular más efectivamente sus proyectos.

En el 1997 se realizó otra convocatoria para solicitar propuestas de las ONG de las diez regiones del Departamento de la Familia, según se había propuesto en el Plan Estratégico de Cinco Años. Después de la evaluación de todas las propuestas sometidas $(\mathrm{N}=68)$, se incorporaron 13 nuevos proyectos a los 38 que continuaron ofreciendo servicios para un total de 51 proyectos de Apoyo a la Familia. Actualmente hay un total de 48 proyectos en nueve regiones del Departamento de la Familia ofreciendo servicios en áreas tan diversas como prevención de embarazos, prevención e intervención con la violencia doméstica, desarrollo de destrezas de crianza, promoción de actividades educativas, recreativas y culturales, prevención proactiva con jóvenes desertores escolares de ambos sexos, apoyo a padres y madres adolescentes, apoyo a mujeres jefas de familia y proyectos de autogestión y cogestión, entre otros.

La Unidad de Asesoría Técnica está llevando a cabo varios estudios de evaluación de efectividad de los servicios que ofrecen los Proyectos de Apoyo a la Familia. Los servicios evaluados hasta el momento son en las áreas de prevención de embarazo, desarrollo de destrezas de crianza, prevención de violencia doméstica y desarrollo de destrezas sociales y prevención de deserción escolar en adolescentes. El estudio de evaluación de resultados de los proyectos que ofrecen servicios para la prevención de embarazos, el único terminado, arroja hallazgos alentadores. El análisis de los datos confirmó que hubo cambios en el conocimiento y la conducta de los jóvenes, además de la comunicación entre padres y adolescentes, como resultado de los servicios ofrecidos.

\section{Conclusiones}

En el desarrollo e implantación de la política social de apoyo y fortalecimiento a las familias en Puerto Rico nos encontramos con la ingente tarea de conocer las necesidades de servicios de apoyo y fortalecimiento de las familias en Puerto Rico, desarrollar un plan de cinco años que recogiera el sentir de "las familias" y otros sectores de 
interés, así como transformar la política social en servicios concretos a dichas familias.

Pronto nos dimos cuenta de lo monumental del esfuerzo y de las controversias al interior del estudio de las necesidades de "las familias" en una arena de reivindicaciones sociales, económicas y políticas. En el proceso entendimos que el desarrollo de la política social de prestación de servicios en el área de apoyo y fortalecimiento de la familia tenía que darse en el contexto de la noción de necesidades como derechos sociales de personas y sectores tales como las mujeres, jóvenes, etc. Nos preguntamos, si uno de los objetivos principales de la legislación de apoyo y fortalecimiento de la familia es salvaguardar la seguridad de todos los miembros de la familia, icómo eliminar o reducir la violencia en nuestras familias si no aseguramos el derecho que tienen las mujeres, los jóvenes y los niños/as a vivir una vida libre de violencia?; ¿cómo ayudar a las mujeres jefas de familias y mujeres sobrevivientes de violencia doméstica si no respondemos a sus necesidades de empleo (con un salario que no sea de subsistencia), vivienda, cuido de niños, transportación, acceso a servicios de salud física y mental, etc.?

Tratando de responder a todas estas interrogantes desarrollamos un modelo de estudio que recogiera las voces de todos los actores interesados. Sin embargo, cuando estábamos en el proceso de análisis nos dimos cuenta de que de nuevo corríamos el riesgo de interpretar las voces de todos los sectores de interés que participaron en el proceso de consulta. ¿Cómo asegurarnos de que las voces de las personas que iban a recibir los servicios tuvieran igual peso que las de los profesionales que participaron en la consulta? ¿Cómo asegurarnos de que las interpretaciones hegemónicas de las necesidades no tuvieran el peso mayor? ¿Cómo rebasar los parámetros de la legislación que venía envuelta en un lenguaje de servicios que explícitamente se dirigían a "familias en riesgo" e implícitamente a "familias problemáticas"?

Reconocimos, entonces, que las necesidades de las mujeres han estado históricamente invisibilizadas bajo el manto de la maternidad y de las instituciones de la familia y el matrimonio, y que sus necesidades y derechos como mujeres han estado atados a su capacidad reproductora. Reconocimos, además que los adolescentes mujeres y 
hombres han sido descualificados y obviados en la provisión de la mayor parte de los servicios sociales en Puerto Rico. Las adolescentes embarazadas han sido identificadas como grupo en necesidad de recibir servicios por su condición de madre y no la de mujeres adolescentes. Entendimos que las madres y padres de adolescentes han sido tradicionalmente culpados por los "problemas" de sus hijos e hijas sin tomar en consideración los factores estructurales y sociales que inciden en estos "problemas". Analizamos de nuevo la legislación y los hallazgos de la consulta (documentos, estudios, vistas públicas, grupos focales, encuestas, etc.) y decidimos privilegiar las voces de los grupos subordinados y las voces de los actores que recibirían los servicios.

De la experiencia de las investigadoras (Valle, Albite, Rosado, 1995) en esta implantación y diseño de política social de apoyo y fortalecimiento a las familias en Puerto Rico, podemos evidenciar que el trabajo social puede contribuir al desarrollo de modelos dirigidos al fortalecimiento de los vínculos comunitarios y al apoderamiento o empoderamiento de los sectores cuyas voces no son generalmente escuchadas o atendidas. Que la acción política en pro de nuestra clientela comienza primeramente con el conocimiento claro del trabajador social de los marcos teóricos e ideológicos que utiliza en su quehacer cotidiano. Es en el análisis riguroso de lo que hace y cómo lo hace que sienta la pauta para luchar por una mayor democratización y justicia social. Todo cuenta en la práctica profesional y la acción política: desde el poder del lenguaje que utilizamos (a fin de cuentas el lenguaje revela ideologías y marcos teóricos); el convencimiento de unos principios que sustenten nuestra ejecutoria; el planteamiento de una hipótesis de trabajo y la acción concertada enmarcada en conocimientos y destrezas desde el escenario y tipo de práctica profesional que desempeñemos. Si creemos en la capacidad de las comunidades, sus familias, mujeres y jóvenes para autogestionarse y en la responsabilidad del Estado de apoyar sus esfuerzos, debemos entonces insertarnos en una red de solidaridad que contribuya a la eliminación de la desigualdad y a la liberación de las personas, familias y comunidades. 


\section{Referencias}

Álvarez, A.R. (1994). El Bienestar Social. En J. Glay Mejía, Ed., Diseño de Proyectos de Bienestar Social. Cali, Colombia: Publicación de ALAETS-CELATS.

Estivill, J. (1985). Los papeles perdidos de las políticas sociales. En Política Socialy Servicios Sociales, Marciega.

Fraser, N. (1989). Unruly Practices, Minn: University of Minnesota Press.

Lechner, N. (1988). Los patios interiores de la democracia: Subjetividad y política, Santiago: FLACSO.

Meenagham, T. y Keith, K. (1994). Policy Analisis and Research Technology. Chicago: Liceum Book, Inc.

Omnibus Reconciliation Act (1993). Título IV-B. Family Support and Family Preservation. Washington: Gobierno Federal.

Pratts, S. (1994). La privatización del pacto social San Juan: EPD Ediciones Porta Coeli.

Santana Rabel, L. Los límites y paradojas del estado benefactor, Punto y coma, vol. 111, no. 1 y 2. Universidad del Sagrado Corazón, pp. 17- 22.

Valle, D.; Albite, L. y Rosado, I. (1995). Estudio de necesidades para el desarrollo de servicios preventivos de apoyo y fortalecimiento de las familias en Puerto Rico. Administración de Familias y Niños, Departamento de la Familia.

Valle, D.; Albite, L. y Rosado, I. (1995). Comprehensive Child and Family Services Plan. Administración de Familias y Niños, Departamento de la Familia. 
